\title{
Hydrodynamic transport properties of hard-sphere dispersions. II. Porous media
}

\author{
R. J. Phillips ${ }^{a)}$ and J. F. Brady \\ Department of Chemical Engineering, California Institute of Technology, Pasadena, California 91125 \\ G. Bossis \\ Laboratoire de Physique de la Matière Condensée, Université de Nice, Parc Valrose, 06034 Nice Cedex, \\ France
}

(Received 8 March 1988; accepted 24 August 1988)

\begin{abstract}
The hydrodynamic transport properties of hard-sphere dispersions are calculated for volume fractions $(\phi)$ spanning the dilute limit up to the fluid-solid transition at $\phi=0.49$. Particle distributions are generated by a Monte Carlo technique and the hydrodynamic interactions are calculated by Stokesian dynamics simulation. The effects of changing the number of particles in the simulation cell are investigated, and the scaling laws for the finite-size effects are determined. The effects of using various levels of approximation in computing both the farand near-field hydrodynamic interactions are also examined. The transport properties associated with porous media-permeabilities and hindered diffusion coefficients-are determined here. The corresponding properties of freely mobile suspensions are determined in a companion paper [Phys. Fluids 31, 3462 (1988)].
\end{abstract}

\section{INTRODUCTION}

In the companion paper (Phillips, Brady, and Bossis'), hereafter referred to as I, we showed how a newly developed method for evaluating hydrodynamic interactions in suspensions could be used to calculate such bulk properties as sedimentation velocities, self-diffusion coefficients, and effective viscosities. The approach we have developed, which is essentially the Stokesian dynamics method of Durlofsky et al. ${ }^{2}$ adapted for application to infinite systems by Brady et al., ${ }^{3}$ can also be used to calculate transport properties of porous media, and in fact some results for such calculations have already been reported for periodic arrays of spheres. ${ }^{3}$

In this paper we present results obtained by applying the method of Paper I to the study of disordered porous media made up of hard spheres. The transport properties we have calculated consist of permeabilities, short-time hindered translational diffusion coefficients, rotational permeabilities or spin viscosities, and short-time hindered rotational diffusion coefficients. As in I, we examine volume fractions $(\phi)$ ranging from infinite dilution to the fluid-solid transition at $\phi=0.49$, and we compare the effects of calculating the hydrodynamic interactions at three levels of approximation. Finally, whenever possible, we compare our results with experiment and with other results obtained from the literature, thereby providing some criteria for assessing the accuracy of our method.

In the past, several researchers have commented on the qualitative differences between the nature of hydrodynamic interactions in free suspensions and in porous media. ${ }^{46}$ Essentially these differences result from the fact that, in porous media, it is necessary to apply external forces (and torques) to particles in order to hold them immobile, whereas in suspensions of neutrally buoyant particles, no such forces need

\footnotetext{
a) Present address: Department of Chemical Engineering, Massachusetts Institute of Technology, Cambridge, Massachusetts 02139.
}

be applied. The effect of these applied forces is to screen the hydrodynamic interactions between particles; causing them to decay as $K / r^{3}$ over long distances, where $K$ is the permeability, rather than decaying as $1 / r$, as is the case for Stokes flow in a free suspension.

Previously developed methods of calculation often incorporate this screening behavior directly by considering interactions within an effective medium made up of particles and fluid, satisfying the so-called Brinkman equation. Examples of this approach include Brinkman's ${ }^{4}$ evaluation and the evaluation of Kim and Russel ${ }^{7}$ of the permeability of porous media, and the calculation of Freed and Muthukumar $^{8}$ of the mobility of a sphere moving through a bed of stationary spheres. The Stokesian dynamics method used here does not rely upon a similar effective medium assumption. It does, however, provide a method that allows one to calculate many-body interactions accurately in the far-field limit, and thus the screening behavior just discussed arises naturally for systems in which the sphere velocities are constrained to be zero. Indeed, Durlofsky and $\mathrm{Brady}^{\mathrm{g}}$ have carefully studied such interactions in porous media and have shown that, using the Stokesian dynamics method, one can observe Brinkman screening. Thus there is ample evidence to show that our method correctly captures the rather complicated nature of long-range hydrodynamic interactions in porous media.

As in I, the results to be presented here were obtained using a Monte Carlo approach. The procedure consists of generating a series of samples, each modeling a portion of the microstructure of a porous medium, calculating the transport property of interest for each sample, and then averaging to obtain values describing the behavior of a macroscopic system. In the following section we briefly describe our calculational methods. This is followed by a presentation of our results; we then conclude by commenting on some apparent differences between the results obtained here and those of Paper I. 


\section{CALCULATIONAL PROCEDURES}

The details of our sample-generating procedures are presented in I, and will not be repeated here. Essentially we generated several samples in which the hard spheres were arranged in a random fashion. For volume fractions less than 0.25 , these samples were generated using random successive addition; that is, $N$ sphere locations were chosen one by one, with each choice being tested for overlap and rejected if overlap occurred. For $0.25<\phi<0.49$, it was more convenient to place the spheres in some configuration and then move them about using a random-stepping routine to ensure that each sample was disordered. The locations of the $N$ spheres were contained within a "periodic box," which was replicated in all directions out to infinity through the application of periodic boundary conditions. This procedure completely specifies the configuration of an infinite system of spheres, thus allowing the evaluation of transport properties to proceed.

We refer the reader to Refs. 2 and 3 for a detailed description of our method for evaluating and summing hydrodynamic interactions, as only a brief summary will be presented here. The relationship between the forces $(F)$, torques $(\mathrm{L})$, and translational and rotational velocities (U $-\langle\mathbf{u}\rangle$ and $\mathbf{\Omega}-\langle\boldsymbol{\omega}\rangle)$ of $N$ spheres interacting under conditions of low particle Reynolds number can be summarized by the following matrix equation:

$$
\left(\begin{array}{l}
\mathbf{F} \\
\mathbf{L}
\end{array}\right)=\left(\begin{array}{ll}
\mathbf{R}_{F U}^{*} & \mathbf{R}_{F \mathbf{R}}^{*} \\
\mathbf{R}_{L U}^{*} & \mathbf{R}_{\mathbf{L} \Omega}^{*}
\end{array}\right) \cdot\left(\begin{array}{l}
\mathbf{U}-\langle\mathbf{u}\rangle \\
\mathbf{\Omega}-\langle\boldsymbol{\omega}\rangle
\end{array}\right) .
$$

Here $\mathrm{F}$ and $\mathrm{L}$ are vectors of dimension $3 N$ giving the force and torque exerted by all $N$ particles on the fluid. Similarly, $\mathrm{U}$ and $\mathbf{\Omega}$ are $3 N$ translational and rotational velocity vectors, respectively. The vectors $\langle u\rangle$ and $\langle\omega\rangle$ are the suspension average-fluid and particle phase average-translational and rotational velocities. The subscripts on the submatrices give the coupling between the various components. As in Paper I, the superscript asterisk implies Ewald sums. The resistance matrix in (1) is the submatrix, given in Eq. (3) of I, of the grand resistance matrix $\mathscr{R}$ [Eq. (2) of I] that includes the effects of a bulk rate of strain. For the properties of interest here, the bulk rate of strain is zero and we have discarded the unnecessary components.

In the Stokesian dynamics formulation, the resistance matrix of (1) is actually the sum of two other matrices, one containing far-field interaction terms and the other containing near-field, lubrication interactions. The far-field resistance matrix accounts for all many-body interactions in a way that is analytically correct in the far-field limit, while lubrication interactions are calculated using a pairwise additivity assumption. The three levels of approximation referred to in the Introduction are related to the level of accuracy used in determining the far-field interactions. If only forces and translational velocities are accounted for in the far-field calculations, thereby neglecting torques and all higher moments of the force density on the particle surfaces, then the resistance matrix reduces to the $3 N \times 3 N$ matrix $\mathbf{R}_{F U}^{*}$, and we have what we call the " $F$ method." Going one step further and accounting for particle torques, including rotation-translation coupling, results in the full resistance matrix shown in (1), and corresponds to the "F-T method." Finally, including particle stresslets, which are induced moments that result from a symmetric distribution of force density on the particle surfaces, results in the most accurate of the three levels of approximation, the "F-T-S method." Note that $\mathbf{R}_{F U}^{*}, \mathbf{R}_{L \Omega}^{*}$, etc., will not be the same for the three levels of approximation, as including torques and/or stresslets will not only change the size of the complete resistance matrix but will also change the individual components of the submatrices listed.

Once having computed our approximation to the true resistance matrix, we are in a position to perform the necessary averages to obtain the transport properties alluded to earlier. The permeability $\mathbf{K}$ of a porous medium is usually defined as the proportionality constant that relates the average pressure gradient and the average velocity for a system in which the particles are fixed in space $(U=0, \Omega=0$, $\langle\omega\rangle=0$ ), i.e., through Darcy's law:

$$
\nabla\langle p\rangle=-\eta \mathbf{K}^{-1} \cdot\langle\mathbf{u}\rangle,
$$

where $\eta$ is the fluid viscosity. The pressure gradient is related to the average force $\langle\mathbf{F}\rangle$ by consideration of an overall force balance, $\nabla\langle p\rangle=-n\langle\mathbf{F}\rangle$ (cf. Brady et al. ${ }^{3}$ ); thus the "resistivity," $K^{-1}$, of a porous medium is given by

$$
\mathbf{K}^{-1}=n\left\langle\mathbf{R}_{F U}^{*}\right\rangle,
$$

where $n$ is the number density of particles. The angle brackets in (3) imply an average over all particles in the resistance matrix and an average over all realizations of the suspension microstructure. For an isotropic, statistically homogeneous hard-sphere microstructure, the permeability becomes a scalar $K$ :

$$
\mathbf{K}=\boldsymbol{K} \mathbf{I} \text {. }
$$

The resistivity $K^{-1}$ is proportional to the average drag on a particle, and may sometimes be referred to as the average drag coefficient.

Equation (3) bears a close relation to the average used to define the sedimentation velocity [cf. Eq. (6) of I]. The sedimentation velocity is given by $\langle\mathbf{U}\rangle=\left\langle\mathbf{R}_{F U}^{*}{ }^{-1}\right\rangle$, while the permeability is given by $\mathbf{K}=\left\langle\mathbf{R}_{F U}^{*}\right\rangle / n$. Apart from the scale factor $n$, it is the commutation of the inversion operator and the average that distinguishes between sedimentation and permeability; for the sedimentation velocity, the resistance matrix is inverted and then averaged, while for the permeability the resistance matrix is averaged and then inverted. In general, these operations do not commute, and the sedimentation velocity and the permeability are quite different. (Commutation is only possible for matrices corresponding to periodic arrays of particles.)

As with the permeability $K$ we define the "rotational permeability" $K_{r}$ as the relation between the average torque $\langle\mathrm{L}\rangle$ and the macroscopic angular velocity $\langle\omega\rangle$ when all the particles are fixed in space ( $U=\boldsymbol{\Omega}=\mathbf{0}$ ). Thus, in analogy with (3), we have

$$
\mathbf{K}_{r}^{-1}=n\left\langle\mathbf{R}_{L \Omega}^{*}\right\rangle .
$$

We see that $\mathbf{K}_{r}$ is also isotropic in the hard-sphere microstructure, and we have

$$
\mathbf{K}_{r}=K_{r} \mathbf{I} \text {. }
$$


The inverse of the rotational permeability is also known as the spin viscosity, which is relevant to ferrofluids. ${ }^{3,10,11}$ Note also that the cross couplings $\left\langle\mathbf{R}_{F}^{*}\right\rangle$ and $\left\langle\mathbf{R}_{L U}^{*}\right\rangle$ are zero in an isotropic medium. If the microstructure were not isotropic, these cross terms would be present and are related to the handedness or chirality of the porous medium. ${ }^{12}$

The calculation of the mobilities, or hindered diffusion coefficients, of individual particles requires a somewhat different procedure than that described above. In this case, instead of setting all of the particle velocities equal to zero, we set $N-1$ particle velocities equal to $\langle\mathbf{u}\rangle(=0)$ and evaluate the relationship between the force and velocity of the freely mobile particle. This relationship is governed by a $3 \times 3 \mathrm{ma}-$ trix that lies along the diagonal of $\mathbf{R}_{F U}^{*}$, and thus the mobility of particle $\alpha$ is given by $\left(\mathbf{R}_{F U}^{* \alpha \alpha}\right)^{-1}$. The mobility averaged over all the particles in the system, along with the StokesEinstein relation, defines the short-time hindered diffusion coefficient, $\mathbf{D}_{0}^{\mathrm{HD}}$,

$$
\mathbf{D}_{0}^{\mathrm{HD}}=k T \overline{\frac{1}{N} \cdot \sum_{\alpha=1}^{N}\left(\mathbf{R}_{F U}^{* \alpha \alpha}\right)^{-1}},
$$

where $k$ is Boltzmann's constant and $T$ is the absolute temperature. The overbar in (7) denotes the average over all realizations of the microstructure. Again, $D_{0}^{H D}$ can be described by a single scalar quantity in (7) $D_{0}^{\mathrm{HD}}$.

Note the distinction between this diffusion coefficient and the short-time self-diffusion coefficient reported in $\mathrm{Pa}$ per I: here we calculate the mobility of a particle surrounded by other particles that are fixed in space (i.e., a porous medium), whereas in Paper I the surrounding particles were mobile and were required to be free of any applied forces or torques (i.e., a free suspension). Just as in Paper I, however, we are restricted to the calculation of short-time coefficients, which describe motion on a time scale so short that the mobile particle does not move a distance comparable to its own radius or to the distance between particle surfaces. The importance of this restriction is readily apparent for the case of a mobile particle "wandering" randomly within a small "cage" formed by immobile particles: clearly the instantaneous mobility or hindered diffusion of the mobile particle will be finite while its long-time hindered diffusivity will be zero. Finally, we again point out that a rotational hindered diffusion coefficient $D_{r}^{\mathrm{HD}}$, analogous to $D_{0}^{\mathrm{HD}}$, can be obtained by simply substituting $\left(\mathbf{R}_{L \Omega}^{* \alpha \alpha}\right)^{-1}$ for $\left(\mathbf{R}_{F U}^{* \alpha \alpha}\right)^{-1}$ in (7).

The calculations required to obtain the results presented in the next section were all performed on a CYBER 205 supercomputer, with the specifics of the time requirements being identical to those given in Paper I. In actual fact, all calculations presented here and in Paper I were calculated simultaneously. Each of the data points reported here represents an average taken over ten samples, a procedure that was sufficient to give us $95 \%$ confidence intervals that were generally within $5 \%$ of the average values reported. Our statistical errors were reduced by the fact that each sample produces three values for each of the transport properties described, a consequence of the independence of those quantities when calculated in each of the three coordinate directions. Thus although only ten samples are represented in each data point, that point is actually an average over 30 values. As normalizations we use the dilute limiting values: $K^{-1}=\frac{9}{2} \phi a^{-2}, K_{r}^{-1}=6 \phi, D_{0}^{\mathrm{HD}}=k T /(6 \pi \eta a)$, and $D_{r}^{\mathrm{HD}}$ $=k T /\left(8 \pi \eta a^{3}\right)$, where $a$ is the sphere radius.

\section{RESULTS AND DISCUSSION}

Our results for the calculations described above can be examined in several ways. First, when possible we shall compare our results with those that have been reported previously in the literature. In making these comparisons we wish to emphasize the flexibility of the Stokesian dynamics method, with which one can calculate all the transport properties of porous media that are reported here, the transport properties of suspensions reported in Paper I (and these two sets of calculations can be done simultaneously), and also perform dynamic simulations if so desired. In addition, we shall compare the effects of changing the size of the periodic boxes that make up the infinite systems by varying the number of particles per periodic box $(N)$ and, as stated previously, we shall discuss results corresponding to three levels of approximation in the hydrodynamic calculations. This latter information should be of interest to those who wish to perform similar calculations, but do not necessarily require the most accurate, and hence most costly, formulation that is available. It also sheds some light on the nature of the hydrodynamic interactions being studied.

\section{A. Permeability or resistivity}

We report our permeability results in the form of the average drag coefficient, $K^{-1}$, as this is the more natural calculation. In Fig. 1(a) we compare our resistivity results for values of $N$ equal to 14, 27, and 64 particles per periodic box with the results of Kim and Russel, ${ }^{7}$ the Carman correlation, ${ }^{13}$ and the results of Zick and Homsy ${ }^{14}$ for a periodic simple cubic lattice of spheres. Note that, in general, the results for random systems lie below those for SC lattices, a fact no doubt related to the larger void spaces through which fluid may flow in disordered media. Also, the Stokesian dynamics results agree well with those of Kim and Russel and the Carman correlation up to volume fractions of about 0.35 , while at higher volume fractions our results tend to underestimate the correct value of $K^{-1}$. This trend is consistent with the results obtained for periodic systems, ${ }^{3}$ and can be explained in a similar fashion.

In the Stokesian dynamics approximation to the resistance matrix, we assume that interactions between nearly touching spheres are dominated by lubrication forces, an assertion that is not valid for the case of a porous medium in which there is no relative motion between the spheres. As is the case for the sedimentation problem discussed in $I$, the fluid flows between the large gaps separating the particles and lubrication forces do not come into play. Thus the lubrication forces included in our hydrodynamic calculations do not significantly affect the drag on a particle, and we are forced to rely solely upon our far-field interactions for that calculation. Not surprisingly, the far-field interaction terms alone underestimate the true drag force experienced by the spheres.

This observation is clearly illustrated in Fig. 1(b), 

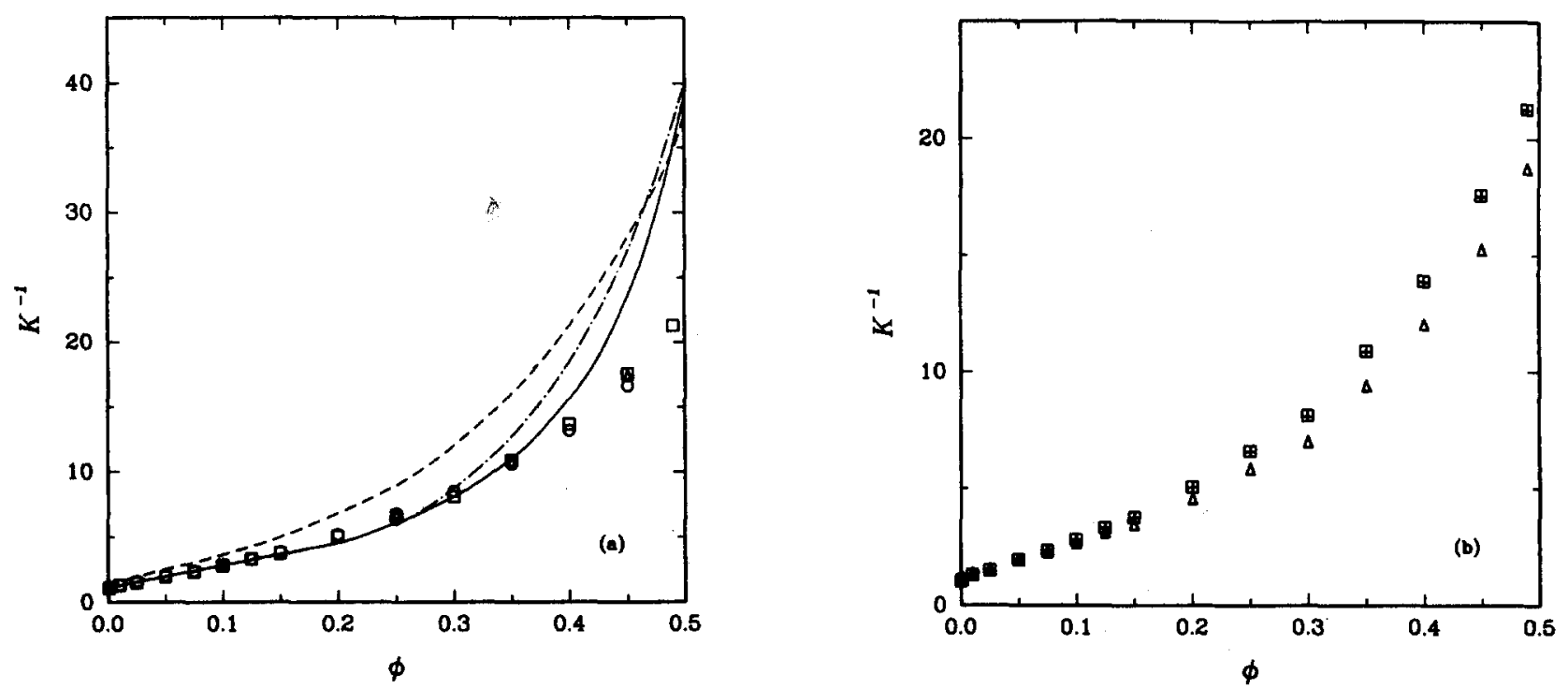

FIG. 1. Comparison of the effects of (a) number of spheres per periodic box $(N)$, and (b) level of approximation in the formation of the resistance matrix for the calculation of the average drag coefficient, $K^{-1}$. The Carman correlation, the results of Kim and Russel for random arrays, and those of Zick and Homsy for periodic simple cubic lattices are also shown. (a) $O, 14$ spheres; $\square, 27$ spheres; $\Delta, 64$ spheres; -, Kim and Russell (1985); - - -, Zick and Homsy (1982); $-\cdot-$ Carman correlations. (b) $\square, 27$ spheres; + , minus lubrication; $\Delta$, minus stresslets.

where it is seen that lubrication forces have virtually no effect on the results. In contrast, including the dipole interaction terms of the moment expansion described by Brady et al. (i.e., moving from the F to the F-T-S method) does significantly improve the accuracy of the calculations. (Including torques only, as in the F-T formulation, did not significantly alter the $F$ method results, and so for simplicity those data were not plotted.) The effect of the stresslet terms is indicative of what would be required to obtain more accurate values of $K^{-1}$ using this method: the moment expansion used in determining the far-field interaction terms would have to be extended to include quadrupole, octupole, and all higher multipole moments, a procedure that would require excessive computer time. Consideration of the behavior of simple cubic arrays shows that to achieve $10 \%$ accuracy at $\phi=0.5$ requires multipoles through the hexadecapole.

One other significant observation evident from Fig. 1 (a) is the absence of periodicity effects, as evidenced by the roughly equivalent results obtained for the three values of $N$ studied. As in Paper I, we attempt to explain this result by comparing the effect of a sphere's periodic reflections, which are arranged in a simple cubic lattice with volume fraction $\phi / N$ as a result of the periodic boundary conditions, with the effect of all the other spheres in the system, which we assume act as a random porous medium. The drag coefficient for a sphere in a dilute, simple cubic lattice is ${ }^{5}$

$$
K_{\mathrm{SC}^{-1}}=1+1.74 \phi^{1 / 3} \text {, }
$$

while for a dilute, fixed, random array we have

$$
K_{\text {ran }}^{-1}=1+(\sqrt{3} / 2) \phi^{1 / 2} \text {. }
$$

Therefore for the effect of the periodic reflections to be negligible we require

$$
1.74(\phi / N)^{1 / 3} /(4.5)^{1 / 2}(1-1 / N)^{1 / 2} \phi^{1 / 2}<1,
$$

or, for large $N$,

$$
N>0.57 \phi^{-1 / 2} \text {. }
$$

The inequality (11) is also the requirement that the Brinkman screening length $(\sqrt{2} / 3) a \phi^{-1 / 2}$ be small compared to the size of the periodic box. As long as this condition is satisfied, the medium will appear disordered rather than ordered, and the finite-size effects will scale as $N^{-1}$ (see below). Except for the most dilute systems studied, the inequality (11) was satisfied in our simulations.

\section{B. Hindered diffusion coefficient}

As stated previously, our calculation of short-time, hindered diffusion coefficients consists of evaluating the mobility of a sphere in a porous medium and applying the StokesEinstein equation. Recall, however, that in our simulations we are not able to calculate the mobility of a single mobile particle, since that particle is periodically replicated throughout space as a result of the periodic boundary conditions. Thus although the Brinkman screening present in porous media significantly reduces the effects of the long-range periodicity, there are $O\left(N^{-1}\right)$ effects that remain. In an unbounded porous medium, when one particle moves, both the average suspension velocity $\langle\mathbf{u}\rangle$ and the average force $\langle\mathbf{F}\rangle$ are zero. Macroscopic mass and momentum balances confirm this. In the periodic case, since we move all images of the test particle, a nonzero average force may be required in order to ensure that $\langle u\rangle=0$. The average velocity must be zero within the periodic cell, and a macroscopic pressure gradient may be needed to pump the fluid in opposition to the motion of the test particle. This effect of periodicity decays as $N^{-1}$ as shown by Durlofsky and Brady. ${ }^{9}$

In Fig. 2(a), our results for 14, 27, and 64 particles per periodic box are shown along with the asymptotic low $\phi$ result derived by Freed and Muthukumar. ${ }^{8}$ Clearly the agreement between the two sets of results is excellent for the 

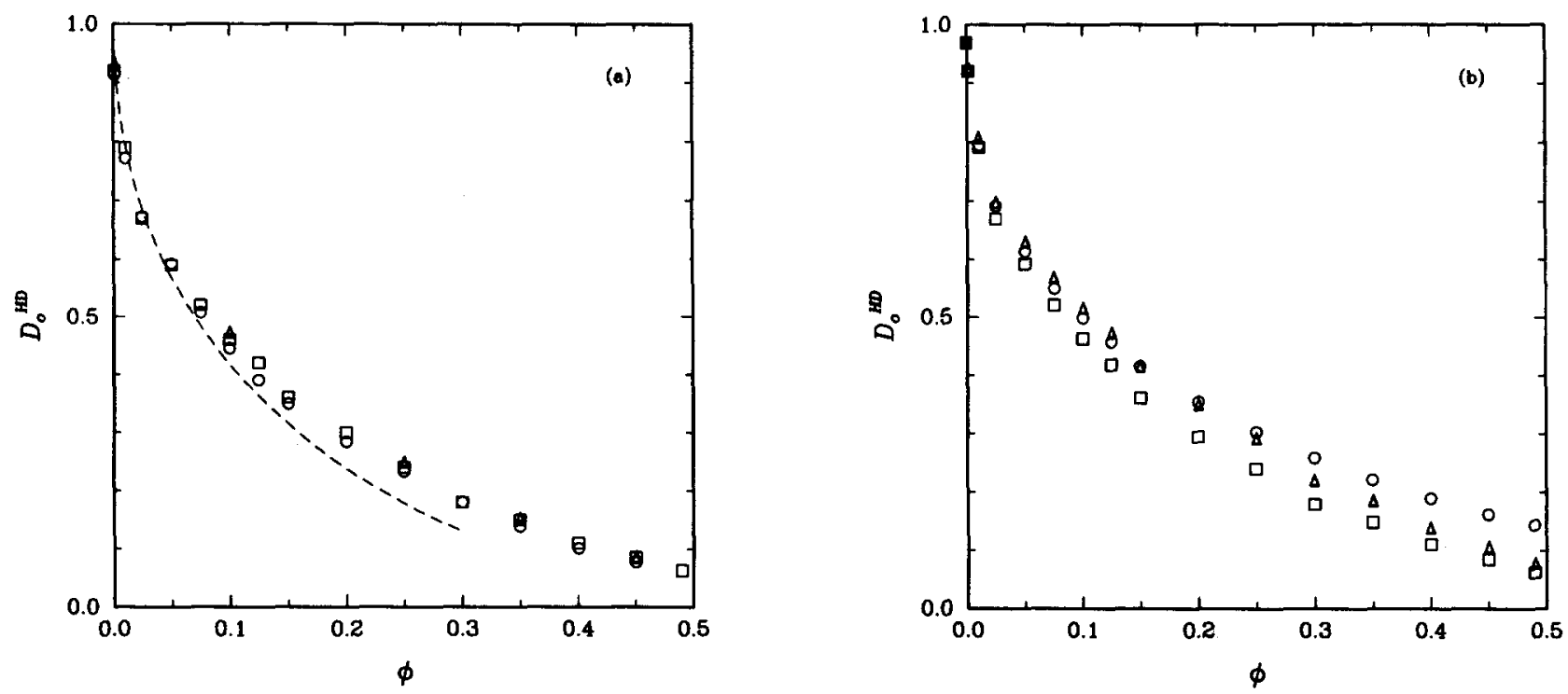

FIG. 2. Comparison of the effects of (a) number of spheres per periodic box ( $N$ ), and (b) level of approximation in the formation of the resistance matrix for the calculation of the short-time hindered diffusion coefficient, $D_{0}^{\text {HD }}$. The result reported by Freed and Muthukumar, valid for low $\phi$, is also presented. (a) $O, 14$ spheres; $\square, 27$ spheres; $\triangle, 64$ spheres; - - , Freed and Muthukumar (1978) (low volume fraction). (b) $\square, 27$ spheres; $O$, minus lubrication; $\triangle$, minus stresslets.

dilute systems where the result obtained by Freed and Muthukumar is correct. At higher $\phi$, the calculations of Freed and Muthukumar seem to underestimate the hindered diffusivity, although they do give the proper trend. Finally, periodicity effects are seen to be negligible for the reasons discussed above.

In Fig. 2(b), the complete F-T-S method results for $N=27$ are shown and are compared with those obtained from the simpler F method and from the F-T-S method without lubrication. As in Figs. 1(a) and 1(b), the results for the F-T method deviate only slightly from those for the $F$ method and so were left out for clarity. The data of Fig. 2(b) can be interpreted as meaning that lubrication and stresslet interactions are of comparable importance up to volume fractions of about 0.25 , while for higher volume fractions lubrication interactions begin to dominate the effects of all the higher-order moments. Because stresslets do seem to be important here over such a wide range of volume fractions, it could be worthwhile to explore possible alternative methods of including them in the formulation. One such approach might be to include stresslets in a pairwise fashion along with lubrication interactions, thereby classifying stresslet contributions as near-field rather than far-field interactions. This would increase the computational efficiency of the method by reducing the size of the mobility matrix that must be inverted as part of the far-field calculations (see Paper I), but would also probably overestimate the effect of the stresslets. (From a rigorous mathematical point of view, the stresslet
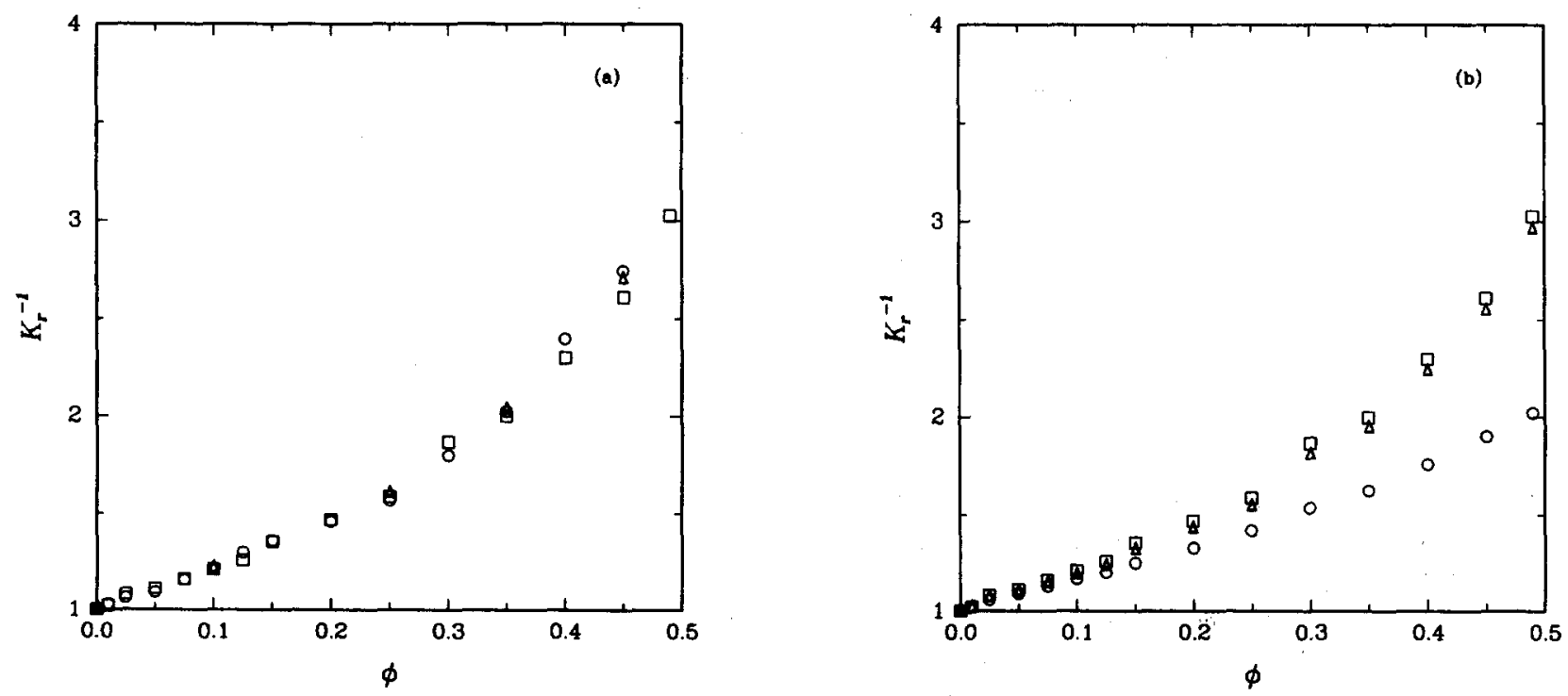

FIG. 3. Comparison of the effects of (a) number of spheres per periodic box ( $N$ ), and (b) level of approximation in the formation of the resistance matrix for the calculation of the rotational resistivity, $K_{r}^{-1}$. (a) Legend same as for 2(a). (b) Legend same as for 2(b). 

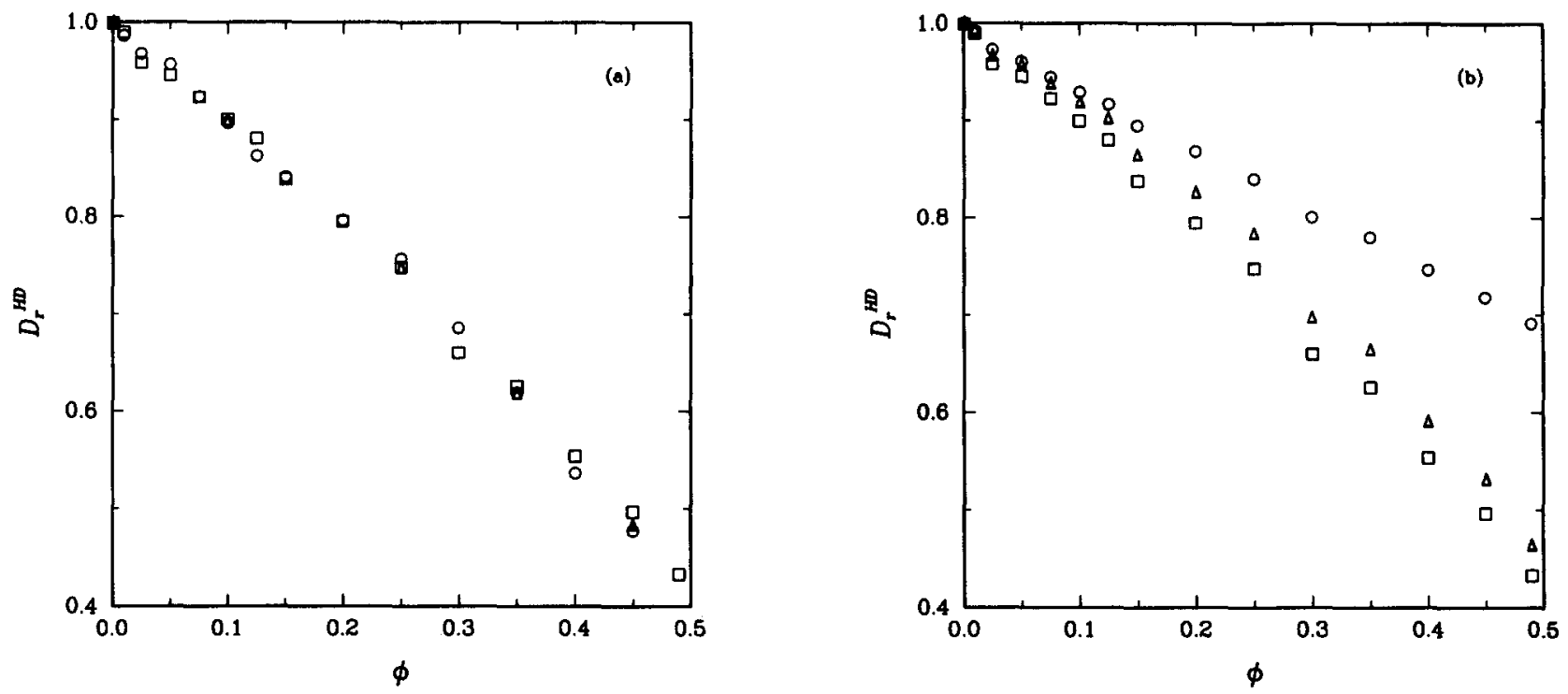

FIG. 4. Comparison of the effects of (a) number of spheres per periodic box $(N)$, and (b) level of approximation in the formation of the resistance matrix for the calculation of the short-time rotational hindered diffusion coefficient, $D_{r}^{\mathrm{HD}}$. (a) Legend is same as for 2(a). (b) Legend is same as for 2(b).

interactions are conditionally convergent and need to be properly summed, so some difficulties with this approach might arise.)

\section{Rotational permeablilty and rotational hindered diffusion coefficient}

We complete our discussion of transport properties of porous media by presenting our results for the rotational analogs of the transport properties just presented. Our results for the rotational resistivity, $K_{r}^{-1}$, are presented in Figs. 3 (a) and 3(b), while the rotational hindered diffusion coefficients are plotted in Figs. 4(a) and 4(b). As was the case for the translational transport properties, neither set of rotational quantities calculated show any effects of periodicity, as the three values of $N$ used all seem to yield essentially identical results. In addition, for both cases the lubrication interaction terms appear to be much more important than the stresslet interaction terms, particularly at the higher volume fractions studied. Note also that, in contrast to the resistivity, lubrication is important for the rotational resistivity as there is now relative motion between particle surfaces. At present we are not aware of any theoretical or experimental results with which to compare our data. It may be remarked, however, that the calculations of Kim and Russel and Freed and Muthukumar can be trivially extended to the rotational case.

\section{CONCLUSIONS}

We have successfully applied the Stokesian dynamics method described by Brady $e t$ al. ${ }^{3}$ to a wide class of problems involving free suspensions and porous media. In Paper I our results for transport properties associated with suspensions of hard spheres, including sedimentation velocities, self-diffusion coefficients, and effective viscosities, were presented and analyzed. In this paper a similar set of results are given for porous media, including calculations of permeabilities and short-time hindered diffusion coefficients. Among the more prominent differences between the results presented in these two papers are the effects incurred by our imposition of periodic boundary conditions. While periodicity effects were clearly important in the translational properties (sedimentation and self-diffusion) of free suspensions, they are not at all apparent in the results presented in this paper. The reasons for this difference lie in Brinkman screening effects, which cause interactions to decay more rapidly in porous media than in suspensions, thereby decreasing any effects of longrange order. As a final point, we wish to state that calculations such as those described here serve as examples of ways in which the Stokesian dynamics method can be applied without actually performing dynamic simulations. Thus the method itself has the potential to be useful in any number of problems that could benefit from the evaluation of manybody, as opposed to pairwise, hydrodynamic interactions.

\section{ACKNOWLEDGMENTS}

We would like to thank our colleague L. Durlofsky for useful discussions on the application of Stokesian dynamics to porous media.

This research was supported in part by National Science Foundation Grants No. CBT-8696067 and No. INT8413695. Computer time was provided by the von Neuman Supercomputer Center. RJP would also like to acknowledge the support of a NSF fellowship.

'R. J. Phillips, J. F. Brady, and G. Bossis, Phys. Fluids 31, 3462 ( 1988 ). ${ }^{2}$ L. Durlofsky, J. F. Brady, and G. Bossis, J. Fluid Mech. 180, 21 (1987). ${ }^{3}$ J. F. Brady, R. J. Phillips, J. C. Lester, and G. Bossis, J. Fluid Mech. 195, 257 (1988).

${ }^{4}$ H. C. Brinkman, Appl. Sci. Res. A 1, 27 (1947).

${ }^{5}$ P. G. Saffman, Stud. Appl. Math. 52, 115 (1973). 
${ }^{6}$ E. J. Hinch, J. Fluid Mech. 83, 695 (1977).

'S. Kim and W. B. Russel, J. Fluid Mech: 154, 269 (1985).

${ }^{8}$ K. F. Freed and M. Muthukumar, J. Chem. Phys. 68, 2088 (1978).

${ }^{9}$ L. Durlofsky and J. F. Brady, Phys. Fluids 30, 3329 (1987).

${ }^{10}$ M. Zuzovsky, P. M. Adler, and H. Brenner, Phys. Fluids 26, 1714 (1983).
"'R. E. Rosensweig, Ferrohydrodynamics (Cambridge, New York, 1987). ${ }^{12}$ D. L. Koch and J. F. Brady, Phys. Fluids 30, 642 (1987).

${ }^{13}$ R. B. Bird, W. E. Stewart, and E. N. Lightfoot, Transport Phenomena (Wiley, New York, 1960).

${ }^{14}$ A. Zick and G. M. Homsy, J. Fluid Mech. 115, 13 (1982). 